Mara MANENTE

Università Ca' Foscari di Venezia

\title{
LES VERBES ALLER ET VENIR ET LEUR INCOMPATIBILITÉ AVEC L'EMPLOI RÉSULTATIF $^{*}$
}

\section{Introduction}

Les verbes aller, venir, arriver, partir, entrer et sortir lexicalisent la composante 'mouvement' et c'est pourquoi ils peuvent être définis comme 'verbes de mouvement'. Intuitivement, le mouvement peut être défini comme un processus dynamique par opposition à un état. L'appellation 'verbes de mouvement' est, toutefois, trop limitée. En effet, ces verbes ne lexicalisent pas seulement la composante 'mouvement' mais aussi la composante 'déplacement' (pour une description typologique de l'événement spatial voir TALMY, 1985). À ce sujet il faut préciser que le déplacement et le mouvement sont deux notions tout à fait différentes : le mouvement ne conduit pas nécessairement à un changement de lieu, tandis que le déplacement l'implique. En particulier, le déplacement dénoté par les verbes aller, venir, arriver, partir, entrer et sortir est délimité, dans le sens qu'il implique une transition à partir d'un point initial (début du déplacement) pour atteindre un point final (fin du déplacement). C'est pourquoi ces verbes sont aussi appelés 'verbes de changement de lieu' (voir LEGENDRE et SORACE, 2003). Contrairement aux verbes arriver, partir, entrer, sortir et venir, qui encodent le but de la transition, le verbe aller n'encode pas le but de la transition mais il le projette en syntaxe. C'est pourquoi les verbes arriver, partir, entrer, sortir et venir sont définis en tant que verbes intrinsèquement téliques

(1), tandis que le verbe aller est défini en tant que verbe compositionnellement télique (2) :

(1) Jean est arrivé/parti/entré/sorti/venu en deux minutes

(2) Jean est allé *(chez elle) en deux minutes

Contrairement aux verbes arriver, partir, entrer et sortir, les verbes aller et venir ne sont pas compatibles avec l'intervalle temporel mesuré par l'adverbial depuis $x$ temps :

(3) Jean est arrivé/parti/entré/sorti depuis deux minutes ${ }^{1}$

(4) *Jean est allé chez elle depuis deux minutes

(5) *Jean est venu depuis deux minutes

\footnotetext{
* Certains passages de cet article ont été tirés du troisième chapitre de ma thèse de doctorat (MANENTE, 2008). Cependant, l'article se présente enrichi en approfondissements qui ne figuraient pas dans ma thèse. Je voudrais remercier Anne Zribi-Hertz, Marie-France Merger et Marie-Christine Jamet pour certains jugements de grammaticalité. Bien entendu, elles ne sont pas responsables des erreurs que ce travail pourrait contenir.

${ }^{1}$ Pour une analyse de la sémantique des verbes arriver, partir, entrer et sortir et de leur emploi résultatif voir aussi BOUCHARD (1995).
} 
J'emploierai ici le terme résultatif pour référer à la valeur aspectuelle [+durative] impliquant un intervalle temporel entre la conclusion de l'événement télique et le moment de l'énonciation $\mathrm{T}_{0}$ (voir aussi NEDJALKOV et JAXONTOV, 1988). En (3), c'est l'adverbial de durée depuis x temps qui mesure l'intervalle de temps entre la conclusion de l'événement télique et $\mathrm{T}_{0}$.

Dans le but de trouver une analyse qui permette d'expliquer l'incompatibilité des verbes aller et venir avec l'emploi résultatif, je formulerai l'hypothèse que cette incompatibilité relève de la nature déictique de ces verbes.

Mes réflexions sont organisées en quatre parties : dans la première section je présenterai l'approche syntaxique de la description des événements élaborée par PUSTEJOVSKY (1991) et je me pencherai aussi sur la différence entre la notion d'état résultant et celle d'état ; dans la deuxième section je montrerai que l'analyse formelle élaborée par PUSTEJOVSKY (1991) ne saisit pas les différences entre l'Aktionsart du verbe aller et celui des verbes venir, arriver, partir, entrer et sortir ; dans la troisième section, j'envisage l'hypothèse que l'incompatibilité des verbes aller et venir avec l'emploi résultatif est due au caractère déictique de ces verbes. Finalement, dans la quatrième partie, j'approfondirai l'analyse, respectivement, de l'emploi duratif-résultatif et de l'emploi duratif-statif des verbes arriver, partir, entrer et sortir.

\section{Le sous-événement $\mathrm{E}_{2}$}

La quadripartition en états, activités, accomplissements et achèvements de l'aspect lexical (Aktionsart) des prédicats verbaux élaborée par VENDLER (1957) est, peut-être, la plus connue parmi les classements que connaît la théorie de l'Aktionsart. Les types d'Aktionsart analysés par VENDLER (1957) peuvent être ainsi définis et résumés :

- les prédicats d'état décrivent une situation qui ne comporte pas de changement dans un intervalle de temps donné (être fatigué, savoir quelque chose, aimer le chocolat, etc.) ;

- les prédicats d'activité décrivent un processus qui se compose d'une suite d'actes identiques dont la somme est conçue comme une seule action (marcher, courir, manger, regarder un tableau, etc.) ;

- les prédicats d'accomplissement décrivent une situation qui comporte un processus avec une transition d'un état vers un autre (manger une pomme, rentrer chez soi, courir un 100 mètres, etc.) ;

- les prédicat d'achèvement décrivent une situation qui comporte, en revanche, une transition instantanée d'un état vers un autre (sursauter, atteindre le sommet, arriver, partir, sortir, entrer, etc.).

VENDLER (1957) range les événements téliques, c'est-à-dire délimités par un point terminal (ou culmination), en deux classes: les achèvements et les accomplissements. Les verbes dénotant un achèvement sont doués d'une télicité lexicale et ils sont donc intrinsèquement téliques. Plus précisément, ils dénotent des événements à déroulement rapide dont le point initial coïncide idéalement avec le point final de la transition.

Toutefois, la télicité peut concerner aussi l'ensemble du prédicat. En d'autres termes, elle peut être compositionnelle outre qu'inhérente au verbe lexical. Si la 
télicité se vérifie au niveau du prédicat, on se trouve face à des accomplissements. La plupart des verbes dénotant des activités est souvent compatible avec un contexte télique, pourvu que le but du processus soit explicitement réalisé ou bien qu'il soit récupérable à partir du contexte discursif. Le verbe aller en (2) décrit donc une transition qui est interprétée comme un accomplissement. En (2), par conséquent, c'est le prédicat verbal qui dénote une transition et non pas le verbe lexical.

À la différence de VENDLER (1957), PUSTEJOVSKY (1991) repère trois types d'Akionsart des prédicats verbaux: les états, les processus (c'est-à-dire les activités) et les transitions (c'est-à-dire les accomplissements et les achèvements). Selon PUSTEJOVSKY (1991), les achèvements et les accomplissements peuvent être représentés de la même manière, c'est-à-dire comme des transitions composées de deux sous-événements $\left\langle\mathrm{E}_{1} ; \mathrm{E}_{2}\right\rangle$ dont la distance temporelle est mesurée par l'adverbial en $x$ temps. Plus précisément, $\mathrm{E}_{1}$ dénote le point initial de la transition, tandis que $\mathrm{E}_{2}$ dénote le point final (ou état résultant) de la transition. Dans l'optique de l'analyse de PUSTEJOVSKY (1991), les verbes arriver, partir, entrer, sortir, venir et aller, en tant que verbes dénotant une transition, ont une structure événementielle du type $\left\langle\mathrm{E}_{1} ; \mathrm{E}_{2}\right\rangle$. Pour ce qui concerne les états et les activités, PUSTEJOVSKY (1991) propose de représenter les premiers par l'entremise d'un seul événement $\langle\mathrm{e}\rangle$ et les deuxièmes par l'entremise de la séquence d'événements identiques $\left\langle e_{1} \ldots e_{n}\right\rangle$. L'analyse de PUSTEJOVSKY (1991) diffère donc de celle de VENDLER (1957) car elle décrit la structure des événements en termes formels et, en même temps, elle parvient à des généralisations concernant la structure événementielle.

\section{1. État résultant $v s$ état}

Dans l'optique de l'analyse de PusteJovsky (1991), l'état issu d'une transition correspond au sous-événement $\mathrm{E}_{2}$.

Or, la notion d'état diffère de celle d'état issu d'une transition. Un état est indépendant de tout processus antérieur, tandis qu'un état issu d'une transition dénote à la fois un état et le résultat de la transition qui a donné lieu à cet état (voir NEDJALKOV et JAXONTOV, 1988). À partir de ces observations, je propose de décrire la différence entre un état et un état issu d'une transition en termes de traits. Plus précisément, j'envisage l'hypothèse qu'un état indépendant de tout processus antérieur est spécifié pour le trait aspectuel [-résultat], tandis qu'un état issu d'une transition, c'est-à-dire un état résultant, est spécifié pour le trait aspectuel [+résultat]. Dans l'optique de mon analyse, le trait [+résultat] est donc associé au sous-événement $\mathrm{E}_{2} \mathrm{~d}$ 'une transition.

Dans l'introduction, j'ai observé que les verbes arriver, partir, entrer et sortir sont compatibles avec l'interprétation résultative impliquant un intervalle temporel duratif entre la conclusion de l'événement télique et le moment de l'énonciation. SCHADEN (2007 : 107) observe que dans l'interprétation résultative «la phase résultante de l'éventualité fournit la phase homogène tenant pendant toute la durée de l'intervalle de 'depuis'». Or, les prédicats d'état en (6), tout comme les prédicats d'achèvement arriver, partir, entrer et sortir (voir (3)), sont compatibles avec l'intervalle de temps duratif mesuré par l'adverbial depuis x temps : 
(6) a. Jean l'aime depuis deux ans

b. Jean est là depuis deux jours

En (6), la phase homogène s'étendant pendant toute la durée de l'intervalle temporel signalé par 'depuis' est fournie par la composante aspectuelle stative inhérente, respectivement, au prédicat d'état (6a) et à la phrase copulativeprédicative (6b). Les prédicats d'état sont donc des prédicats duratifs qui s'étendent d'une façon homogène dans un intervalle de temps donné.

En exploitant l'analyse formelle de PUSTEJOVSKY (1991), je propose qu'avec les prédicats d'achèvement l'aspect duratif mesuré par l'adverbial depuis x temps réfère à l'intervalle temporel entre l'état résultant $\left\langle\mathrm{E}_{2}\right\rangle$ et $\mathrm{T}_{0}$, tandis qu'avec les prédicats d'état l'aspect duratif réfère à l'intervalle temporel entre l'événement $<e>$ dénotant l'état et $\mathrm{T}_{0}$. Dans le premier cas (voir (7)), on aura une interprétation durative-résultative, tandis que dans le deuxième cas (voir (8)), on aura une interprétation durative-stative :

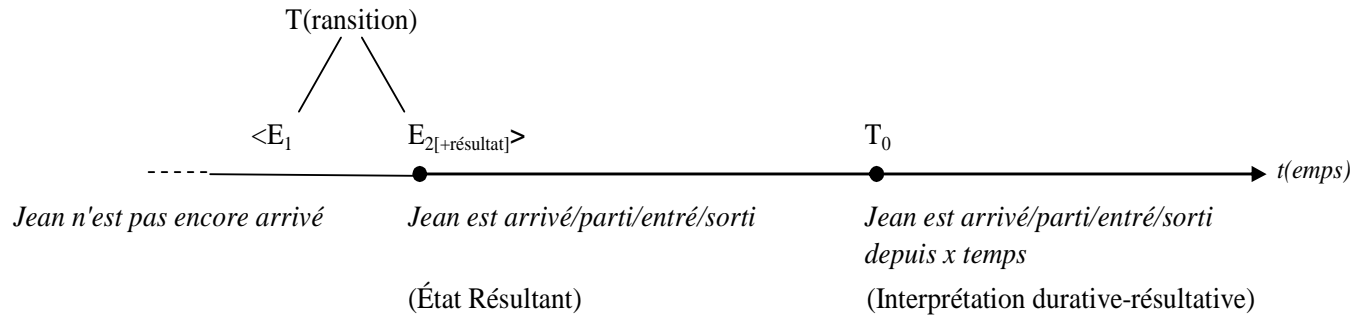

(8)

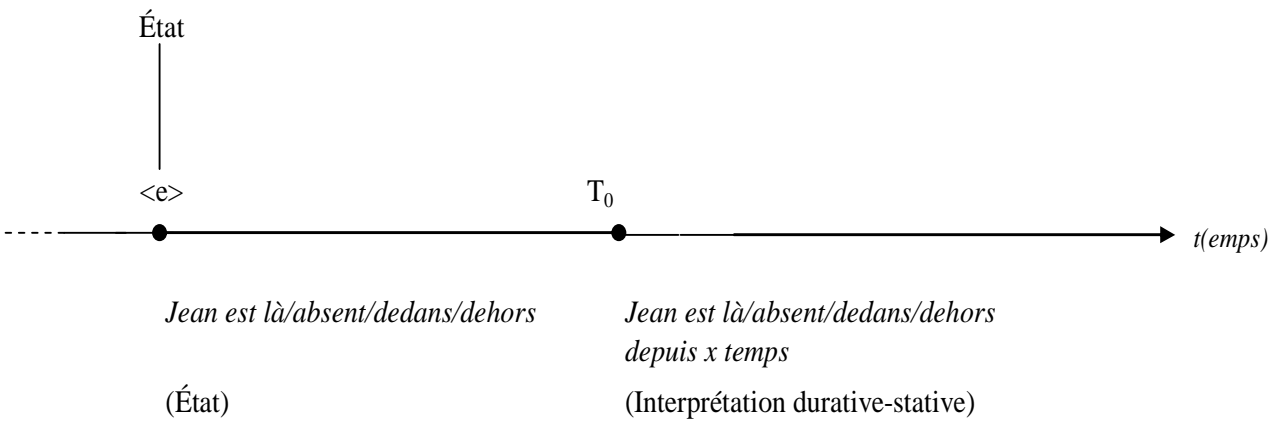

L'intervalle temporel duratif en (7) doit être calculé à partir de $\mathrm{T}_{0}$ jusqu'à un moment du passé dont la localisation temporelle coïncide avec la conclusion (état résultant) de la transition dénotée par le verbe. En revanche, l'intervalle temporel 
duratif en (8) se calcule à partir de $\mathrm{T}_{0}$ et s'étend jusqu'à un moment du passé qui coïncide avec le début de l'événement dénoté par le prédicat d'état. Ces observations portent à admettre que l'état résultant d'une transition est toujours compatible avec l'interprétation résultative. Toutefois, cette conclusion entraîne un problème car l'état résultant des verbes aller et venir n'est pas compatible avec une interprétation résultative.

Bien que certains auteurs (CREISSELS, 2000 et LAGAE, 2005) aient observé que l'incompatibilité des verbes aller et venir avec l'intervalle de temps duratif mesuré par depuis $x$ temps relève de l'Aktionsart de ces verbes, aucune de ces analyses ne se révèle satisfaisante. En particulier, dans son analyse des formes verbales en être+participe passé, CREISSELS (2000) affirme qu'un verbe est compatible avec l'interprétation résultative seulement s'il est intrinsèquement télique. Autrement dit, selon CREISSELS (2000), la compatibilité avec l'interprétation résultative n'est possible qu'en liaison avec une interprétation télique du sens lexical (Aktionsart) du verbe. C'est pourquoi, selon l'auteur, le verbe aller, en tant que verbe compositionnellement télique, n'a pas d'emploi résultatif. Toutefois, il s'avère que le verbe venir, qui est intrinsèquement télique, n'a pas d'emploi résultatif. La généralisation formulée par CREISSELS (2000) n'est donc pas suffisamment prouvée.

Le modèle élaboré par PUSTEJOVSKY (1991) ne parvient pas non plus à expliquer l'incompatibilité de aller et venir avec l'intervalle temporel mesuré par depuis x temps. Dans l'optique de l'analyse de PUSTEJOVSKY (1991), les verbes venir et aller, en tant que verbes dénotant une transition, sont associés à une structure événementielle du type $\left\langle\mathrm{E}_{1} ; \mathrm{E}_{2}\right\rangle$ (voir supra). En d'autres termes, PUSTEJOVSKY (1991) décrit l'aspect de l'événement dénoté par les verbes aller et venir de la même manière que l'aspect de l'événement dénoté par les verbes arriver, partir, entrer et sortir. La représentation formelle élaborée par PUSTEJOVSKY (1991) se révèle, toutefois, trop générale car elle ne parvient pas à cercler le facteur qui entraîne l'incompatibilité des verbes aller et venir avec l'interprétation résultative. Afin de délimiter avec précision le facteur responsable de cette incompatibilité, je me pencherai, dans les pages suivantes, sur l'analyse de l'aspect lexical inhérent à ces verbes.

\section{L'Aktionsart des verbes aller et venir}

Plutôt que de représenter l'aspect lexical inhérent au verbe aller, j'envisage l'hypothèse que la séquence $\left\langle\mathrm{E}_{1} ; \mathrm{E}_{2}\right\rangle$ représente l'événement télique qui se vérifie au niveau du prédicat verbal aller+SP. Posant que le verbe aller est compositionnellement télique (voir (2)), CuMMINS (1996: 71) affirme que aller est un verbe de mouvement qui dénote un processus plutôt qu'une transition ${ }^{2}$. Contrairement à ce qu'affirme CUMMINs (1996), je ne vois pas très bien comment l'aspect lexical inhérent au verbe aller peut dénoter une activité. Plus précisément, la question se pose de savoir si, à l'intérieur du modèle formel élaboré par PUSTEJOVSKY (1991), l'Aktionsart du verbe aller peut être représenté par

\footnotetext{
${ }^{2}$ «Aller is a verb of motion but it is a process rather than a transition » (CUMMINS, $\left.1996: 71\right)$.
} 
l'entremise d'un seul événement $\left\langle\mathrm{e}_{1} \ldots \mathrm{e}_{\mathrm{n}}\right\rangle$ conçu comme une activité. Dans les pages qui suivent, je répondrai par la négative en justifiant ma démarche.

Comme j'ai observé dans l'introduction, le verbe aller, tout comme les verbes arriver, partir, entrer, sortir et venir, lexicalise deux composantes sémantiques, à savoir le 'mouvement' et le 'déplacement'. Tous les verbes d'activité impliquent le mouvement et certains parmi eux lexicalisent aussi la composante 'déplacement' ${ }^{3}$. Or, l'analyse du verbe aller en tant que verbe d'activité soulève un problème. En effet, contrairement aux verbes d'activité, qui décrivent des processus non délimités, l'événement dénoté par le verbe aller doit être délimité afin d'interpréter correctement le sens du verbe (voir (2)). Mieux, par rapport aux verbes d'activité, le verbe aller encode la composante 'déplacement orienté', c'est-à-dire une direction par rapport à un lieu d'arrivée ${ }^{4}$. À la lumière de ces observations, il s'ensuit que le verbe aller ne peut pas être défini comme un verbe d'activité car un verbe d'activité n'entraîne pas statutairement un déplacement orienté. Par conséquent, contrairement à ce que soutient CuMMINS (1996), l'Aktionsart du verbe aller ne peut pas être représenté comme une activité $\left\langle\mathrm{e}_{1} \ldots \mathrm{e}_{\mathrm{n}}\right\rangle$. En outre, posant que le verbe aller n'encode pas le but de la transition, l'Aktionsart de aller ne peut même pas être représenté comme une transition $\left\langle\mathrm{E}_{1}\right.$; $\mathrm{E}_{2}>$.

À la lumière de ces observations, je propose de représenter l'aspect lexical inhérent au verbe aller par l'entremise de deux positions événementielles dont la première est remplie par le sous-événement $\left\langle\mathrm{E}_{1}\right\rangle$, tandis que la deuxième est vide $<$ _> et correspond à la composante 'déplacement orienté' :

\section{(9) aller}

$<\mathrm{E}_{1} ;{ }_{-}>$

Étant donné le caractère compositionnellement télique du verbe aller, j'envisage l'hypothèse que le sous-événement $\mathrm{E}_{2}$ dénotant le but de la transition est donc projeté au niveau du prédicat et non pas du verbe :

$$
\begin{aligned}
& \text { (10) aller } \\
& <\mathrm{E}_{1} ;{ }_{-}>*\left(\mathrm{E}_{2[+ \text { résultat }]}\right)
\end{aligned}
$$

En revanche, pour ce qui concerne le verbe intrinsèquement télique venir, le sous-évènement $\mathrm{E}_{2}$ est projeté au niveau du verbe lexical :

$$
\begin{aligned}
& \text { (11) venir } \\
& <\mathrm{E}_{1} ; \mathrm{E}_{2[+ \text { résultat }]}>
\end{aligned}
$$

\footnotetext{
${ }^{3}$ Comme je l'ai remarqué dans l'introduction, la notion de mouvement diffère de celle de déplacement. Parmi les prédicats dénotant une activité, il y en a certains qui encodent la composante 'déplacement' outre que la composante 'mouvement' comme, par exemple, les verbes courir, marcher, nager, etc.

${ }^{4}$ Un 'déplacement orienté' implique donc la présence d'une trajectoire le long de laquelle se déroule l'événement à partir d'une source (point initial du trajet/début du déplacement) pour atteindre un but (point final du trajet/fin du déplacement).
} 
Bien que la représentation formelle en (10) permette de distinguer l'Aktionsart du verbe aller de celui du verbe venir en (11), il s'avère que les représentations en (10) et en (11) n'arrivent pas à saisir le facteur qui empêche l'emploi résultatif de aller et de venir. En effet, la séquence $\left\langle\mathrm{E}_{1} ; \mathrm{E}_{2[+r e ́ s u l t a t]}\right\rangle$ ne décrit pas seulement l'Aktionsart du verbe venir, mais elle décrit aussi l'Aktionsart des verbes arriver, partir, entrer et sortir qui se différencient de venir parce qu'ils sont compatibles avec un emploi résultatif :

\section{(12) arriver, partir, entrer, sortir$$
<\mathrm{E}_{1} ; \mathrm{E}_{[2+\text { résultat }]}>
$$

Ces observations portent à admettre que l'incompatibilité de aller et venir avec l'emploi résultatif ne relève pas de l'Aktionsart de ces verbes.

Dans ce qui suit, je montrerai que l'incompatibilité de aller et venir avec l'interprétation résultative est à ramener à la composante déictique de ces verbes.

\section{Le rôle de la deixis}

Parmi les nombreuses définitions de deixis possibles, je retiendrai la suivante : « la deixis est un procédé d'assignation de la référence qui met en relation le discours avec la situation d'énonciation » (ZRIBI-HERTZ, 1990: 603)

Trois paramètres essentiels doivent être satisfaits afin d'interpréter correctement la relation entre le discours et la situation d'énonciation : l'ego, l'hic et le nunc. L'ego renvoie à l'identité du locuteur, tandis que l'hic et le nunc réfèrent, dans l'ordre, au temps et au lieu où le locuteur prononce l'énoncé. La connaissance de l'identité des participants à l'acte communicatif et leur situation dans le temps et dans l'espace au moment de l'énonciation est une condition nécessaire afin d'interpréter correctement les expressions déictiques.

Les verbes aller et venir sont correctement interprétés seulement si les paramètres gouvernant la deixis sont satisfaits. C'est pourquoi ils sont couramment définis comme verbes déictiques. Plus précisément, d'un point de vue sémantique, aller et venir dénotent une trajectoire qui met en relation les participants à l'acte communicatif avec leur situation dans l'espace au moment de l'énonciation. En particulier, le verbe venir dénote une trajectoire centripète, tandis que le verbe aller dénote une trajectoire centrifuge par rapport au locuteur. L'événement décrit par ces verbes se déroule le long de cette trajectoire et le but de la transition est atteint une fois que le sujet a parcouru la trajectoire et qu'il est arrivé jusqu'au bout de ce parcours. À la lumière de ces observations, je propose d'interpréter l'intervalle entre $\mathrm{E}_{1}$ et $\mathrm{E}_{2}$ (voir (13) et (14)) comme un intervalle déictique qui met en relation les participants à l'acte communicatif avec leur situation dans l'espace au moment de l'énonciation :

$$
\begin{aligned}
& \text { (13) aller } \\
& {\left[<\mathrm{E}_{1} ;{ }_{-}>\mathrm{E}_{2+\text { résultat }}\right]_{\text {déictique }}} \\
& \text { (14) venir } \\
& {\left[<\mathrm{E}_{1} ; \mathrm{E}_{2+\text { résultat }}>\right]_{\text {déictique }}}
\end{aligned}
$$


Comme le montrent (13) et (14), une fois que le but de la transition est atteint, le trait [+résultat] est satisfait. Il en est de même avec les verbes intrinsèquement téliques arriver, partir, entrer et sortir (voir (12)).

Étant donné que le trait [+résultat] doit être satisfait afin de pouvoir interpréter correctement les verbes aller et venir, il s'ensuit que l'incompatibilité de aller et venir avec l'emploi résultatif n'est pas à ramener à ce trait spécifique. Or, posant que aller et venir sont des verbes déictiques et que les paramètres gouvernant la deixis (ego, hic et nunc) sont mis en relation à l'intérieur de l'intervalle compris entre $\mathrm{E}_{1}$ et $\mathrm{E}_{2}$, il s'ensuit que aller et venir peuvent être correctement interprétés seulement à l'intérieur de cet intervalle. Il s'avère donc que le résultat de la transition dénotée par ces verbes ne peut pas s'étendre au dehors de la conclusion de l'événement télique $\left(\mathrm{E}_{2}\right)$ et qu'il ne peut donc pas déclencher d'interprétation résultative.

L'ensemble des observations formulées ci-dessus conduisent donc à admettre que c'est la composante déictique inhérente aux verbes aller et venir qui empêche leur emploi résultatif.

Dans ce qui suit, je me pencherai à nouveau sur l'interprétation durativerésultative et sur l'interprétation durative-stative des verbes dénotant une transition dans le but de montrer que l'interprétation durative-résultative n'est pas déclenchée par la composante [+résultat], mais, au contraire, par la composante [+état] qui est inhérente à l'état résultant.

\section{Interprétation durative-résultative vs interprétation durative-stative}

Les formes en être + participe passé des verbes arriver, partir, entrer et sortir en (15) sont compatibles aussi bien avec une interprétation durative-résultative, où la forme en être+participe passé est interprétée comme le résultat de la transition, qu'avec une interprétation durative-stative, où la forme en être+participe passé est interprétée comme un prédicat d'état :
a. Jean est arrivé
b. Jean est parti
c. Jean est entré
d. Jean est sorti

Un contexte pragmatique convenable permet de mettre en évidence l'interprétation durative-stative des formes en être+participe passé en (15). En (16B), par exemple, les formes en être+participe passé sont employées comme autant de réponses à des questions totales (oui/non) correspondant à des phrases copulatives-prédicatives :
a. A : Est-ce qu'il est là, Jean ?
B : Oui, il est arrivé (= là)/Non, il n'est pas arrivé (= là)
b. A : Est-ce que Jean est absent ?
B : Oui, il est parti (= absent)/Non, il n'est pas parti (= absent)
c. A : Est-ce que Jean est dedans ? 
B : Oui, il est entré (= dedans)/Non, il n'est pas entré (= dedans)

d. A : Est-ce que Jean est dehors ?

B : Oui, il est sorti (= dehors)/Non, il n'est pas sorti (= dehors)

En (16B), le participe passé n'est pas interprété comme l'état résultant de l'éventualité dénotée par arriver, partir, entrer et sortir, mais il est interprété comme un état. Les phrases en (16B) ont, respectivement, le sens de 'Jean est là en ce moment', 'Jean est absent en ce moment', 'Jean est dedans en ce moment', 'Jean est dehors en ce moment'. La compatibilité des participes passés en (16B) avec l'interprétation durative-stative est donc fournie par la composante stative (là, absent, dedans, dehors) inhérente aux verbes lexicaux.

Comme le montrent les phrases en (17), l'adverbial de durée depuis $x$ temps est compatible avec l'interprétation durative-stative du participe passé de ces verbes. Dans ce cas, l'adverbial depuis $x$ temps mesure l'intervalle temporel entre l'événement <e> dénotant l'état et le moment de l'énonciation $\mathrm{T}_{0}$ (voir aussi (8)) :

a. A : Est-ce qu'il est là, Jean ?

B : Oui, il est arrivé (= là) depuis deux jours

b. A : Est-ce que Jean est absent ?

B : Oui, il est parti (= absent) depuis deux jours

c. A : Est-ce que Jean est dedans ?

B : Oui, il est entré (= dedans) depuis deux jours

d. A : Est-ce que Jean est dehors ?

B : Oui, il est sorti (= dehors) depuis deux jours

À la différence des questions en (16A), les questions totales en (18A) correspondent à des phrases en être+participe passé :

a. A : Est-ce qu'il est arrivé, Jean ?

$B$ : Oui, il est arrivé ( $\neq$ là)

B': Oui, il est là

b. A : Est-ce qu'il est parti, Jean ?

B : Oui, il est parti ( $\neq$ absent)

B': Oui, il est absent

c. A : Est-ce qu'il est entré, Jean ?

B : Oui, il est entré ( $\neq$ dedans)

B': Oui, il est dedans

d. A : Est-ce qu'il est sorti, Jean ?

B : Oui, il est sorti ( $\neq$ dehors)

B': Oui, il est dehors

Étant donné que les formes en être+participe passé des verbes arriver, partir, entrer et sortir oscillent entre une interprétation durative-résultative, où la forme en être+participe passé est interprétée comme le résultat de la transition, et une interprétation durative-stative, où la forme en être+participe passé est interprétée 
comme un prédicat d'état (voir (15)), il s'ensuit que les questions en (18A) peuvent être interprétées aussi bien d'une façon résultative que d'une façon stative. Par conséquent, les réponses aux questions en (18A) peuvent être de deux types. Elles peuvent correspondre à des phrases en être+participe passé, qui sont compatibles avec une interprétation durative-résultative (voir (18B)), ou bien elles peuvent correspondre à des phrases copulatives-prédicatives associées à une interprétation durative-stative (voir (18B')).

L'adverbial de durée depuis $x$ temps est compatible avec l'interprétation durative-résultative du participe passé de ces verbes. Dans ce cas, l'adverbial depuis $x$ temps mesure l'intervalle temporel entre l'état résultant $\left\langle\mathrm{E}_{2}\right\rangle$ de l'événement télique et le moment de l'énonciation $\mathrm{T}_{0}$ (voir aussi (7)) :
a. A : Est-ce qu'il est arrivé, Jean ?
B : Oui, il est arrivé ( $\neq$ là) depuis deux jours
b. A : Est-ce qu'il est parti, Jean?
B : Oui, il est parti ( $\neq$ absent $)$ depuis deux jours
c. A : Est-ce qu'il est entré, Jean ?
B : Oui, il est entré ( $\neq$ dedans) depuis deux jours
d. A : Est-ce qu'il est sorti, Jean ?
B : Oui, il est sorti ( $\neq$ dehors) depuis deux jours

Les phrases résultatives en (19B) ont, dans l'ordre, le sens de 'Jean est arrivé il y a deux jours et il est encore là', 'Jean est parti il y a deux jours et il est encore absent', 'Jean est entré il y a deux jours et il est encore dedans', 'Jean est sorti il y a deux jours et il est encore dehors'.

L'interprétation durative -résultative des formes en être+participe passé des verbes arriver, partir, entrer et sortir est légitimée par la composante [+résultat] inhérente aux participes passés (voir (18B) et (19B)), tandis que l'interprétation durative-stative de ces formes est légitimée par la composante [+état] inhérente aux participes passés (voir (16B) et (17B)).

Or, bien qu'un état résultant diffère d'un état, en ce sens qu'un état résultant encode la composante [+résultat], tandis qu'un état tout court ne l'encode pas, il s'avère qu'un état résultant et un état partagent la composante [+état] qui marque l'aspect duratif (voir 1.1.).

À la lumière de ces observations, il s'ensuit que c'est la composante [+état] inhérente au participe passé des verbes arriver, partir, entrer et sortir qui déclenche la compatibilité de ces verbes aussi bien avec l'interprétation durativestative - voir (16B) et (17B) - qu'avec l'interprétation durative-résultative - voir (18B) et (19B).

L'ensemble des données reportées en (16), (17), (18) et (19) conduit à conclure que l'adverbial de durée depuis $x$ temps n'est pas un test assez restrictif de résultativité car il est compatible aussi bien avec l'interprétation durativestative qu'avec l'interprétation durative-résultative des formes participiales en (15). En outre, l'adverbial depuis $x$ temps est aussi compatible avec des interprétations aspectuelles autres que la durative (stative (17) ou résultative (19)), 
notamment l'interprétation perfective (20) et l'interprétation imperfective (21) (voir SCHADEN, $2007: 89)^{5}$ :

(20) Jean a mangé trois pommes depuis minuit (interprétation perfective)

(21) Jean a mangé sans interruption depuis minuit (interprétation imperfective)

Pour ce qui concerne les verbes aller et venir, j'ai observé qu'ils sont incompatibles avec l'emploi résultatif (voir (4)). Or, étant donné que l'interprétation résultative est durative, et que les verbes aller et venir, à cause de leur nature déictique, ne sont pas compatibles avec un intervalle de temps duratif qui s'étend à partir de la conclusion de l'événement télique jusqu'à $\mathrm{T}_{0}$, il s'ensuit que aller et venir ne peuvent pas non plus être interprétés comme des prédicats d'état car les prédicats d'état ont une valeur aspectuelle [+durative]. L'emploi du verbe aller en (22) n'a donc pas le sens de 'Jean est arrivé il y a deux minutes chez elle et il est encore là-bas à $\mathrm{T}_{0}$ '. Il en est de même avec le verbe venir. L'emploi du verbe venir en (23) n'a pas le sens de 'Jean est arrivé il y a deux minutes et il est encore là'. Autrement dit, comme l'observe BERTHONNEAU (1993 : 40), l'emploi de (23) ne permet pas de dire à la fois 'arriver il y a deux minutes' et 'être encore là à $\mathrm{T}_{0}$ ' :

(22) *Jean est allé chez elle depuis deux minutes

(23) *Jean est venu depuis deux minutes

Toutefois, il est possible de lever l'agrammaticalité de l'emploi résultatif en (22) et en (23) en manipulant la structure informationnelle de la phrase, ce qui permettrait de créer un contexte perfectif. Dans ce cas, l'adverbial depuis x temps se place hors de l'intonation d'assertion (voir aussi BERTHONNEAU, 1993 à ce propos) et l'allocuteur est obligé de rechercher, dans le contexte énonciatif, le moment auquel renvoie le processus dénoté par le verbe et de le situer à l'intérieur de l'intervalle temporel dénoté par depuis x temps. Dans les phrases en (24) et en (25) la virgule indique que l'adverbial depuis $x$ temps se place hors de l'intonation d'assertion et que les phrases ont donc une interprétation perfective :

(24) Jean est allé chez elle, depuis deux minutes (interprétation perfective)

(25) Jean est venu, depuis deux minutes (interprétation perfective)

\footnotetext{
${ }^{5}$ L'aspect perfectif dénote un processus borné et accompli qui, dans le cas de (20), a eu lieu dans le passé. En revanche, l'aspect imperfectif dénote un procès sans borne temporelle. En (21) l'adverbial sans interruption marque l'aspect imperfectif (voir SMITH, 1991 pour plus de détails sur les différents types d'aspect).
} 


\section{Conclusion}

Cette analyse prend son point de départ des résultats obtenus par PUSTEJOVSKY (1991) qui ne parviennent pas à saisir les différences entre l'aspect lexical inhérent du verbe aller et celui du verbe venir. Plus précisément, mon analyse a voulu mettre en évidence que la séquence événementielle $\left\langle\mathrm{E}_{1} ; \mathrm{E}_{2}\right\rangle$, employée par PUSTEJOVSKY (1991) pour décrire l'aspect des verbes dénotant une transition, ne permet pas de décrire correctement l'Aktionsart du verbe aller. Contrairement aux affirmations de CuMMINS (1996), mon analyse a mis aussi en évidence que le verbe aller ne peut pas non plus être représenté par la séquence événementielle $\left\langle\mathrm{e}_{1} \ldots \mathrm{e}_{\mathrm{n}}\right\rangle$ dénotant une activité.

Étant donné que le trait [+résultat] doit être satisfait afin de pouvoir interpréter correctement les verbes aller et venir, il m'a semblé légitime de soutenir que l'incompatibilité de aller et venir avec l'emploi résultatif n'est pas à ramener au trait [+résultat]. À partir de ces observations, j'ai proposé que l'incompatibilité de aller et venir avec l'interprétation résultative relève de la composante déictique des ces verbes. Plus précisément, j'ai soutenu que la composante déictique des verbes aller et venir empêche le trait [+résultat] de s'étendre du sous-événement $\mathrm{E}_{2}$, dénotant la conclusion de l'événement télique, jusqu'au moment de l'énonciation $\mathrm{T}_{0}$.

J'ai ensuite montré que l'adverbial depuis $x$ temps ne représente pas un test assez restrictif de résultativité car il ne permet pas de distinguer l'interprétation durative-résultative de l'interprétation durative-stative. En outre, j'ai montré que l'adverbial depuis $x$ temps est aussi compatible avec des interprétations aspectuelles autres que la durative.

\section{BIBLIOGRAPHIE}

BERTHONNEAU Anne-Marie (1993), Depuis vs Il y a que, référence temporelle vs cohésion discursive ou A quoi sert que dans il y a que ?, Le temps, de la phrase au texte, VeTTERS Carl (ed. par), Lille, Presses Universitaires de Lille, p. 9-83.

BOUCHARD Denis (1995), The semantics of syntax: a minimalist approach to grammar, Chicago, The University of Chicago.

CREISSELS Denis (2000), L'emploi résultatif de être+participe passé en français, Passé et Parfait (Cahiers Chronos ; 6), Benninger Céline, CARLIER Anne, LAGAE Véronique (ed. par), Amsterdam, Rodopi, p. 133-142.

Cummins Sarah (1996), Meaning and Mapping, Thèse de doctorat, University of Toronto.

LAGAE Véronique (2005), Les formes en être + participe passé à valeur résultative dans le système verbal français, Temporalité et Attitude (Cahiers Chronos ; 12), MolendiJK Arie, Vet Co (ed. par), Amsterdam, Rodopi, p. 125-142.

LEGENDRE Géraldine, SORACE Antonella (2003), Auxiliaires et intransitivité en français et dans les langues romanes, Les langues romanes, GODARD Danielle (ed. par), Paris, CNRS éditions, p. 185-233.

MANENTE Mara (2008), L'aspect, les auxiliaires 'être' et 'avoir' et l'Hypothèse Inaccusative dans une perspective comparative français/italien, Thèse de 
doctorat, Université Ca' Foscari de Venise et Université de Paris VIII Vincennes Saint-Denis.

NedJalkov Vladimir P., JAXONTOV Sergej J. (1988), The typology of resultative constructions, Typology of resultative constructions, NEDJALKOV Vladimir P. (éd. par), Amsterdam/Philadelphia, John Benjamins Publishing Company, p. 3-61.

PUSTEJOVSKY James (1991), The syntax of event structure, Cognition, Lausanne, Elsevier, 41, p. 47-81.

SCHADEN Gerhard (2007), La sémantique du Parfait. Etude des «temps composés » dans un choix de langues germaniques et romanes, Thèse de doctorat, Université de Paris VIII Vincennes Saint-Denis.

SMith Carlota (1991), The Parameter of Aspect, Dordrecht, Kluwer Academic Publishers.

TALMY Leonard (1985), Lexicalization patterns: semantic structure in lexical forms, Language typology and syntactic description, SHOPEN Timothy (ed. par), Cambridge, Cambridge University Press, vol. III, p. 57-149.

Vendler Zeno (1957), Verbs and Times, The Philosophical Review, Cornell, Cornell University Press, 66, 2, p. 143-160.

ZRIBI-HERTZ Anne (1992), De la deixis à l'anaphore : quelques jalons, La deixis, MOREL Mary-Annick, DANON-BOILEAU Laurent (ed. par), Paris, Presses Universitaires de France. 


\section{Abstract}

This paper deals with the French verbs aller 'to go' and venir 'to come' and their incompatibility with the resultative interpretation. Aller and venir are motion verbs denoting a transition. According to PUSTEJOVSKY (1991), verbs denoting a transition have the event structure $\left\langle\mathrm{E}_{1} ; \mathrm{E}_{2}\right\rangle$.

In this paper, I argue that this representation is unable to capture the inherent Aktionsart properties of aller and venir. I further argue that this representation fails to account for the fact that the verbs aller and venir disallow the resultative interpretation. In contrast with the verbs arriver, partir, entrer and sortir, whose resultant state is compatible with the resultative interpretation, the resultant state of aller and venir doesn't trigger this interpretation. I argue that this restriction derives from the deictic nature of these verbs.

Finally, the adverbial depuis $x$ temps, literally 'since $\mathrm{x}$ time', fails to test durative aspect since it is compatible with both perfective and imperfective interpretations. 